\title{
Association of Serum Homocysteine Level with Migraine in Adults
}

\author{
ISLAM MM ${ }^{1}$, HABIB MA ${ }^{2}$, ISLAM MR ${ }^{3}$, RAHMAN HZ ${ }^{4}$, RIZVI AN ${ }^{5}$, KHAN RK $^{6}$, BHUIYAN MM $^{7}$, \\ AHTESAM MS ${ }^{8}$, ISLAM MF ${ }^{9}$, SALEHIN MF ${ }^{10}$, RAKUNUZZAMAN $M^{11}$, HANNAN MA $^{12}$
}

\begin{abstract}
:
Background: Migraine is the second most common primary headache disorder that has close link to the neurovascular system. The exact pathogenesis of migraine is still not fully understood but several possible theories have been proposed. Hyperhomocysteinemia is one of the coincidental factors whose association with migraine is yet in obscure. Methods: This case control study was conducted in the department of Neurology, Bangabandhu Sheikh Mujib Medical University, Dhaka. A total of 65 patients, who were diagnosed as migraine (with aura or without aura) according to ICHD-3 criteria, were considered as case group and another 65 patients (age and sex matched) with headache other than migraine were considered as control group. Serum homocysteine levels were estimated for both groups and other relevant investigations were done in selective cases. Comparison of serum homocysteine levels between two groups were done to see association of serum homocysteine level with migraine in adults. Results: A total of 50 women and 15 men with mean age of $31( \pm 10.41)$ years and 50 women and 15 men with mean age of $33( \pm 10.91)$ years constituted case and control groups, respectively. The mean $( \pm S D)$ serum homocysteine level in case group $10.71( \pm 4.16) \mathrm{imol} / \mathrm{L}$ was significantly higher than control group $7.62( \pm 2.26) \mathrm{imol} / \mathrm{L},(P$ $<0.001)$. The mean value of serum homocysteine level in migraine without aura (MWOA) patients 11.87 ( \pm 4.18$)$ imol/L was found significantly higher than migraine with aura (MWA) patients $8.23( \pm 1.51)$ imol/L, $(p<0.05)$. There was no significant correlation between severity of migraine headache and frequency of migraine attack with serum homocysteine level. Conclusion: Serum homocysteine level was found significantly higher in migraineurs than non-migraineurs.
\end{abstract}

Key words: Homocysteine, Migraine, Hyperhomocysteinemia, Aura etc.

\section{Introduction:}

Headache is the most common symptom in neurology and very common symptom in other systemic diseases. Headache is defined as pain or any kind of discomfort in the head excluding the lower part of the face and including the upper part of the neck. The pain arises from the pain sensitive structure in the head e.g. from blood vessels, venous sinuses, meninges mostly from basal dura, nerve roots, scalp, orbital contents, Para nasal sinuses, teeth, gum etc. as all have pain receptors, These receptors when stimulated either by

1. Dr. Md Monirul Islam, Resident Neurology, Department of Neurology, BSMMU, Dhaka, Bangladesh.

2. Dr. Md. Ahsan Habib,Associate Professor, Department of Neurology, BSMMU, Dhaka, Bangladesh

3. Dr. Md Rafiqul Islam, Professor and Chairman, Department of Neurology, BSMMU, Dhaka, Bangladesh

4. Dr. Hasan Zahirur Rahman, Professor, Department of Neurology, BSMMU, Dhaka, Bangladesh

5. Dr. Abu Nasir Rizvi, Professor, Department of Neurology, BSMMU, Dhaka, Bangladesh

6. Dr. Rezaul Karim Khan, Professor, Department of Neurology, BSMMU, Dhaka, Bangladesh.

7. Dr. Md. Moniruzzaman Bhuiyan, Professor, Department of Neurology, BSMMU, Dhaka, Bangladesh.

8. Dr. Mohammad Saifullah Ahtesam, Resident Neurology, Department of Neurology, BSMMU, Dhaka, Bangladesh

9. Dr. Mohammad Fakrul Islam, Medical officer, Department of Neurology, Dhaka Medical Colleege, Dhaka, Bangladesh

10. Dr. Mushfequl Salehin, Medical officer, Department of Neurology, Bagura Medical Colleege, Dhaka, Bangladesh

11. Dr. Md Rakunuzzaman, Resident Neurology, Department of Neurology, BSMMU, Dhaka, Bangladesh

12. Dr. M A Hannan, Professor, Department of Neurology, BSMMU, Dhaka, Bangladesh 
distension, traction, compression or irritation the pain sensation starts \& then goes to thalamus when we perceive only pain not the nature but when goes to sensory cortex then we can identify the nature of pain ${ }^{1}$.Most headaches are due to primary headache disorders like tension type headache, migraine and cluster headache. Migraine is the second most common cause of headache and headache related disability in the world ${ }^{2}$. Epidemiological studies have documented its high prevalence and high socio-economic and personal impacts. In the Global Burden of Disease Survey 2010 , it was ranked as the third most prevalent disorder and seventh-highest specific cause of disability worldwide ${ }^{3}$.

Migraine is an episodic primary headache disorder characterized by recurrent attacks of various combinations of headache with neurological, gastrointestinal and autonomic symptoms such as mood change, fatigue, yawning, neck stiffness, polyuria, gastrointestinal disturbance, and a variety of visual, somatic sensory and cognitive phenomena ${ }^{4}$. Migraine is a complex neurological disorder characterized by debilitating headaches and a variety of additional symptoms such as nausea, vomiting, photophobia, phonophobia, occasional sensory disturbances, and other conditions caused by neurological dysfunction in varying combinations ${ }^{5}$. It is a highly prevalent disorder characterized by periodic, commonly unilateral, often pulsatile headaches that begin in childhood, adolescence or early adult life and recur with diminishing frequency during advancing years ${ }^{6}$. The lifetime prevalence of migraine is about $33 \%$ in women and $13 \%$ in $\mathrm{men}^{7}$. The tension type headache was the commonest type $(71.13 \%)$ followed by migraine $(26.05 \%)$ and there was a significant preponderance of females (M:F=1:2) of all the subtypes of migraine found with the study done in Bangladeshi populations ${ }^{8}$. However, the presentation diversity of migraine is complex; two common clinical syndromes associated with migrainous headache can be identified easily that are migraine with aura and migraine without aura. Migraine with aura is called classical or neurologic migraine and migraine without aura is known as common migraine. The ratio of classical to common migraine is $1: 5^{6}$.
The exact pathogenesis of migraine is still not fully understood but several possible theories have been proposed, "The trigemino-vascular theory of Moskowitz"9 is one of those. According to the trigemino-vascular theory, the activation of the trigemino-vascular system (TGVS) leads to release of vasoactive neuropeptides contained in their peripheral nerve endings, especially the calcitonin gene-related peptide (CGRP), production of reactive oxygen species (ROS) and homocysteine. Homocysteine causes migraine headache attack through inhibition of GABA-A receptor, activation of NMDA receptor, neurogenic inflammation and oxidative stress ${ }^{10}$.

\section{Materials \& Methods:}

Patients with migraine headache (with Aura and without Aura) according to ICHD-3 criteria, age more than 18 years were enrolled as case group. Age and sex matched headache (other than migraine) patients, age more than 18 years were selected as control group. Patients who were younger than 18 years, pregnant and breast feeding mother, on vitamin B6, B9 and B12 supplementation, taking drugs which interfere with serum homocysteine level such as Anticoagulant, NSAIDs, Antiplatelet agents, Alcohol, Diuretics, Steroids, Estrogen, Methotrexate and other antiFolate drugs. A semi-structured questionnaire was developed in English. Approval from the Institutional Review Board (IRB) of BSMMU was obtained prior to the commencement of this study. The aims and objectives of the study along with its procedure, risks and benefits were explained to the respondents in easily understandable local language and informed written consent was taken from each. After taking proper history, physical and neurological examination, serum homocysteine level and other relevant investigations were done. Proper diagnosis and treatment were ensured for each person of both groups. For serum homocysteine level, 3cc of whole blood sample was collected from antecubital vein after asepsis. The serum homocysteine level was estimated using photospectrometry technique by Architect plus ci 4100 auto analyzer system in the Department of Biochemistry, BSMMU, Dhaka, Bangladesh. 


\section{Statistical analysis}

At the end of data collection, the frequency of homocysteine were determined for both case and control groups. Then, the mean and standard deviation of serum levels of homocysteine of both case and control groups were calculated. Quantitative data were analyzed by unpaired sample t-test, comparison of two percentages (proportion) between two groups were done by Ztest of proportion and qualitative data were analyzed by $\chi^{2}$ test. To see the correlation of severity and frequency of migraine with homocysteine, Spearman's rank correlation coefficient test and box plot diagram were used. At $95 \%$ Confidence Interval $P$ value $<0.05$ was considered as significant. All statistical analyses were done by SPSS software windows version 22 .

\section{Results and Observations:}

This case control study was carried out in the Headache Clinic, Inpatient and Outpatient Department of Neurology, Bangabandhu Sheikh Mujib Medical University, Dhaka. Patients, who were diagnosed as migraine (with aura or without aura) according to ICHD-3 criteria, were considered as case group and patients with headache other than migraine were considered as control group. $A$ total 130 patients were recruited as study population, of them 65 patients were grouped as case and another 65 patients (age and sex match) were as control.

\section{Table-I}

Distribution of the study groups by gender $(n=130)$

\begin{tabular}{llcl}
\hline Gender & \multicolumn{2}{c}{ Groups } & P-Value \\
\cline { 2 - 3 } & Case (\%) & Control (\%) & \\
\hline Male & $15(23.1)$ & $15(23.1)$ & $1.000^{*}$ ns \\
Female & $50(76.9)$ & $50(76.9)$ & \\
\hline Total & $65(100)$ & $65(100)$ & \\
\hline
\end{tabular}

ns $=$ non-significant, ${ }^{*} \mathrm{P}$ - value was derived from Chi square test

Gender distribution showed that both in case and control group, $76.9 \%$ were female and $23.1 \%$ were male.
Family history of migraine headache

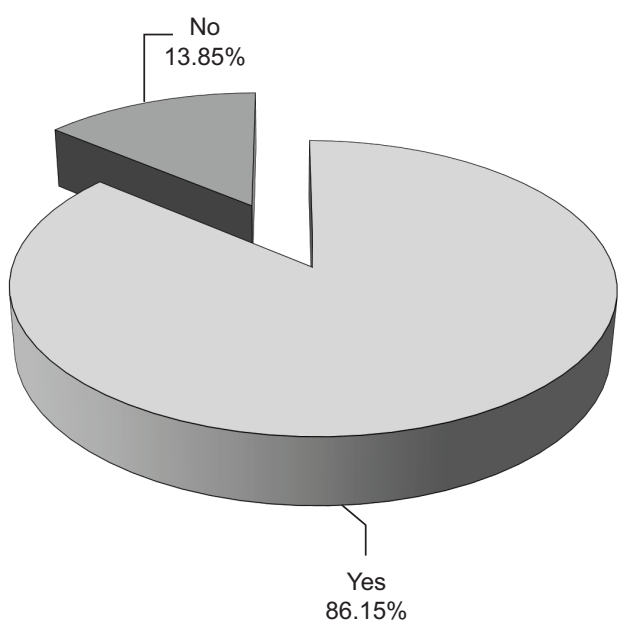

Fig.-1: Pie charts showing family history of migraine in patients

This figure exploded pie chart representing the percentage of patient having family history of migraine $(86.15 \%)$ and $13.85 \%$ having no family history of migraine.

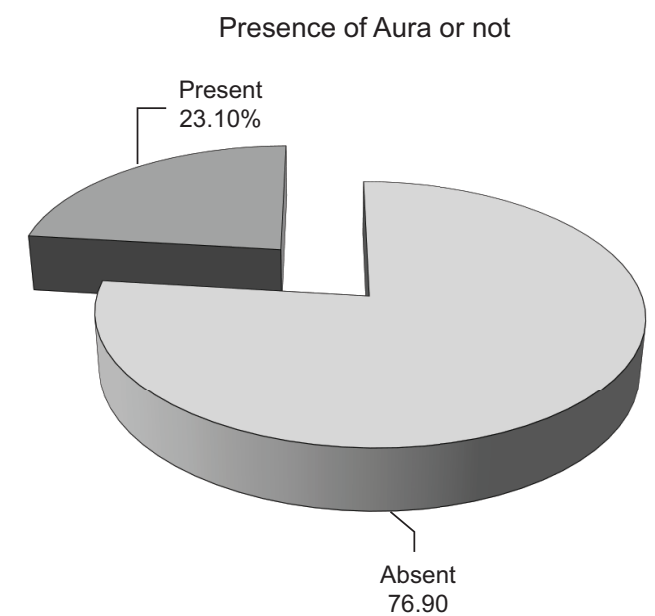

Fig.-2: Pie chart showing distribution of migraine patients according to aura

Piechart shows that a major portion of migraine patients were without aura(76.90\%). Only $23.10 \%$ migraine patients gave history of experiencing aura. 
Table-II

The frequency of serum homocysteine level in both case and control groups

\begin{tabular}{lccc}
\hline Serum homocysteine level $(\mu \mathrm{mol} / \mathrm{L})$ & \multicolumn{2}{c}{ Groups } & P-Value \\
\cline { 2 - 3 } & Case $(\%) \mathrm{n}=65$ & Control $(\%) \mathrm{n}=65$ & \\
\hline $0-5$ & $0(0.0)$ & $6(9.2)$ & $0.0148^{*} \mathrm{~s}$ \\
$5-10$ & $30(46.2)$ & $48(73.8)$ & $0.0020^{*} \mathrm{~s}$ \\
$10-15$ & $30(46.2)$ & $11(16.9)$ & $0.0006^{*} \mathrm{~s}$ \\
$>15$ & $5(7.7)$ & $0(0.0)$ & $0.0258^{*} \mathrm{~s}$ \\
Mean \pm SD & $10.71 \pm 4.16$ & $7.62 \pm 2.26$ & $0.001^{*} \mathrm{~s}$ \\
\hline
\end{tabular}

$\mathrm{s}=$ significant, ${ }^{*} \mathrm{p}$ - value was derived from proportion test, ${ }^{*}{ }^{*} \mathrm{p}$ - value was derived from independent sample $\mathrm{t}$ test.

Table II shows case versus control serum homocysteine level, in between 10-15 $\mu \mathrm{mol} / \mathrm{L} 30$ (46.2)\%vs 11(16.9\%); in between 5-10 $\mathrm{mol} / \mathrm{L} 30$ $(46.2 \%)$ vs $48(73.8 \%)$ and only $5(7.7 \%)$ cases had hyperhomocysteinemia. The mean $( \pm S D)$ value of serum homocysteine level in migraine patients $10.71( \pm 4.16)$ was found increased than in control group $7.62( \pm 2.26)$ which was statistically significant $(p<0.05)$.

This figure 3 shows correlation between headache types and serum homocysteine level both in case and control groups. As headache type is ordinal variable and serum homocysteine level is quantitative variable, so Spearman's rank Correlation coefficient test was done. Here we found positive correlation co-efficient $\left(r_{s}=0.464\right)$

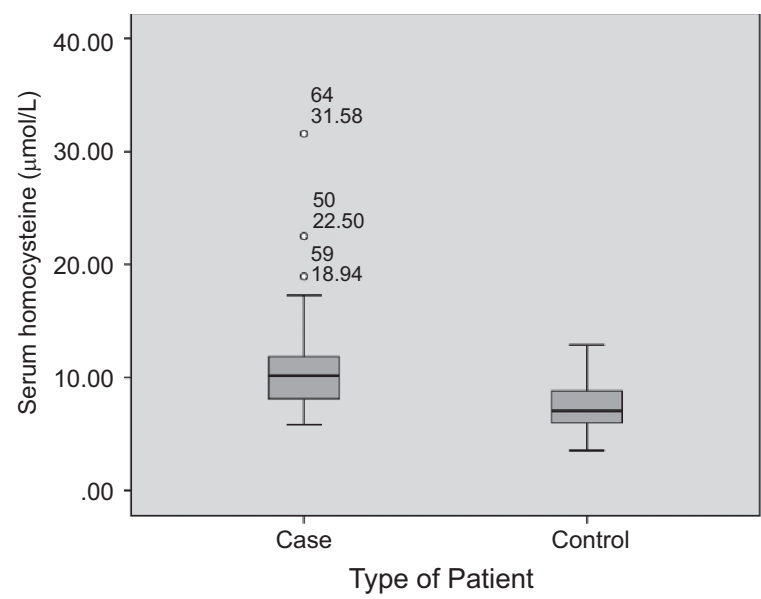

Fig.-3: Box plot diagram showing correlation between headache types and serum homocysteine level (in both groups).

Table-III

Mean of serum homocysteine levels by gender both case \& control group

\begin{tabular}{lcccc}
\hline Gender & $\begin{array}{c}\text { Serum homocysteine } \\
\text { level }(\mu \mathrm{mol} / \mathrm{L})\end{array}$ & Case & Control & P value \\
\hline Female $(\mathrm{n}=50)$ & Mean \pm SD & $9.57 \pm 2.73$ & $6.87 \pm 1.54$ & $<0.001$ ठ \\
Male $(\mathrm{n}=15)$ & Mean \pm SD & $14.51 \pm 5.74$ & $10.14 \pm 2.49$ & 0.017 ठ \\
\hline
\end{tabular}

$\mathrm{s}=$ significant, * $\mathrm{p}$ - value was derived from independent sample $\mathrm{t}$ test.

Table-IV

Mean of serum homocysteine levels among the patients with aura and patients without aura (in case group).

\begin{tabular}{lccc}
\hline $\begin{array}{l}\text { Serum homocysteine } \\
\text { Level }(\mu \mathrm{mol} / \mathrm{L})\end{array}$ & $\begin{array}{l}\text { Migraine with } \\
\text { aura }(n=15)\end{array}$ & $\begin{array}{c}\text { Migraine without } \\
\text { aura }(\mathrm{n}=50)\end{array}$ & P valueð \\
\hline Mean $\pm \mathrm{SD}$ & $8.23 \pm 1.51$ & $11.87 \pm 4.18$ & $0.0018 \mathrm{~s}$ \\
\hline
\end{tabular}

$\mathrm{s}=$ significant, ${ }^{*} \mathrm{p}$ - value was derived from independent sample $\mathrm{t}$ test. 
which was statistically significant $(p<0.05)$. Therefore, we can conclude that headache is significantly correlated with serum homocysteine level.

Table III shows the mean value of serum homocysteine level in female migraine patients $9.57( \pm 2.73) \mu \mathrm{mol} / \mathrm{L}$ was found increased than control group6.87 $( \pm 1.54) \mu \mathrm{mol} / \mathrm{L}$ which was statistically significant $(p<0.05)$. The mean value of serum homocysteine level in male migraine patients $14.51( \pm 5.74) \mu \mathrm{mol} / \mathrm{L}$ was found increased than control group $10.14( \pm 2.49) \mu \mathrm{mol} / \mathrm{L}$ which was statistically significant $(p<0.05)$.

Table IV shows the mean value of serum homocysteine level in migraine patients without aura $11.87( \pm 4.18)$ was found increased than in migraine patients with aura $(8.23 \pm 1.51)$ which was statistically significant $(p<0.05)$.

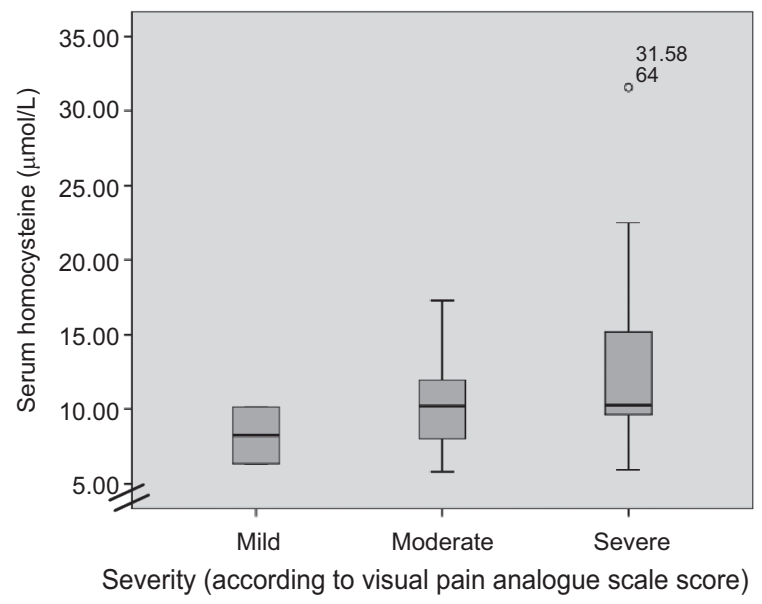

Fig.-4: Box plot diagram showing correlation between severity of migraine headache (according to VAS score) and serum homocysteine level of migraine patients.

This figure 4 shows correlation between migraine headache severity (according to VAS score) and serum homocysteine level of migraine patients. Spearman's rank Correlation coefficient test was done. Here we found positive correlation coefficient $\left(r_{s}=0.144\right)$ which is not statistically significant $(p>0.05)$.

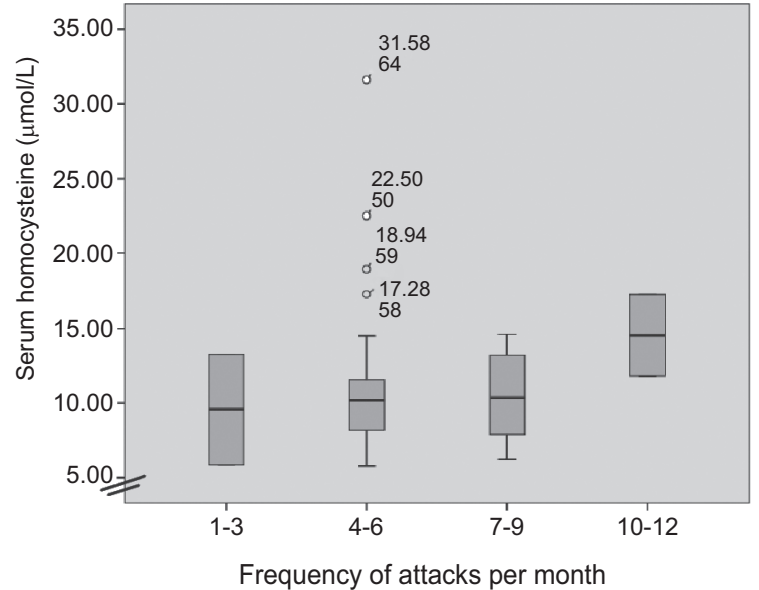

Fig.-5: Box plot diagram showing correlation between frequency of migraine headache attack per month and serum homocysteine level of migraine patients

This figure 5 shows correlation between frequency of migraine headache attack per month and serum homocysteine level of migraine patients. Spearman' srank correlation coefficient test was done. Here we found positive correlation coefficient $\left(r_{s}=0.124\right)$ which is not statistically significant $p>0.05)$.

\section{Discussion:}

Homocysteine, as a highly reactive amino acid, is known to produce endothelial injury via impaired nitric oxide (NO) release that leads to important alterations in vascular function and the coagulant features of the blood. Therefore, homocysteinerelated endothelial dysfunction may be included in the initiation and maintenance of a migraine episode. In our study, the mean ( $\pm S D$ ) serum homocysteine level in migraine patients was found significantly increased than control group [10.71 $( \pm 4.16) \mu \mathrm{mol} / \mathrm{L}$ vs. $7.62( \pm 2.26) \mu \mathrm{mol} / \mathrm{L},(p<0.05)]$. Gavganiand Hoseninian ${ }^{11}$ found significantly higher mean serum homocysteine level in migraine patients than control group $[14.49( \pm 5.03) \mu \mathrm{mol} /$ Lvs $10.92( \pm 4.68) \mu \mathrm{mol} / \mathrm{L}, \mathrm{p}<0.001]$ which was similar to our study. We divided the both case and control group into male and female subgroup for separate statistical analysis. The mean value of serum homocysteine level in female migraine patients $9.57( \pm 2.73) \mu \mathrm{mol} / \mathrm{L}$ was found significantly 
increased than female control group $6.87( \pm 1.54)$ $\mu \mathrm{mol} / \mathrm{L}$. Gavgani and Hoseninian ${ }^{11}$ found significantly higher mean serum homocysteine level in female migraine patients than female control group $[14.50( \pm 5.51)$ vs $11.16( \pm 4.46) \mu \mathrm{mol} /$ L] which coincides with our study. The mean value of serum homocysteine level in male migraine patients $14.51( \pm 5.74) \mu \mathrm{mol} / \mathrm{L}$ was found significantly increased than male control group $10.14( \pm 2.49) \mu \mathrm{mol} / L$. Gavgani and Hoseninian ${ }^{11}$ found statistically significantly higher mean serum homocysteine level in male migraine patients than male control group $[14.48( \pm 4.26)$ vs $10.59( \pm 5.45)$ $\mu \mathrm{mol} / \mathrm{L}$ which coincides with our study. These results may show the increasing importance of homocysteinein migraine etiopathology and it is possible that the different levels of homocysteine are due to ethnic differences and also dietary habit.

Hyperhomocysteinemia is an independent and graded cardiovascular risk factor. Homocysteine did not affect the expression of endothelial nitric oxide synthase (eNOS), but it stimulated formation of superoxide anions leading to neuronal inflammation and vascular damage ${ }^{12}$. About $20-$ $40 \%$ of strokes in women with migraine seem to develop directly from a migraine attack. The prevalence of hyperhomocysteinemia was $7.69 \%$ in patients with migraine. Pizza ${ }^{13}$ conducted a study and found the prevalence of hyperhomocysteinemia $29.16 \%$ in patient with migraine which was not similar to our study, may be due to ethnic difference. These incidences of stroke may be due to high level of serum homocysteine which causes both the events.

The elevated serum homocysteine level causes primarily endothelial cell injury, trigeminal cell firing, and alterations in the coagulant features of blood. Inflammation in the meninges and dilatation of cerebral vessels are thought to cause the pain associated with migraine, which leads to spontaneous trigeminal cell firing. Thus, homocysteine dysfunction can increase the tendency to develop migraine.

Oxidative damage to the vascular endothelium by way of formation of superoxide anions may also increase the probability of migraine and other vascular disorders such as stroke, cardiovascular diseases, vasculitis ${ }^{5}$. The mean value of serum homocysteine level patients migraine without aura $11.87( \pm 4.18) \mu \mathrm{mol} / \mathrm{L}$ was found increased than patients migraine with aura $8.23( \pm 1.51) \mu \mathrm{mol} / \mathrm{L}$ which was statistically significant. Bokharietal (14)found plasma homocysteine level was significantly associated with migraine without aura (MWOA) and plasma homocysteine level was lower inpatients migraine with aura (MWA) than in patients migraine without aura (MWOA) which was similar with our study.

As we found significantly high level of serum homocysteine level in migraine patients, so we also observed the correlation between serum homocysteine level with migraine headache, headache severity and frequency of migraine attack per month. Where, we found significant positive correlation between for serum homocysteine level and migraine headache but statistically non-significant positive correlation between for serum homocysteine level with migraine headache severity and frequency of migraine attack per month, $(p>0.05)$. Significant relationship between serum levels of homocysteine and characteristics of migraine attacks such as severity, frequency and duration were not found in studies conducted by Sadeghiet al., ${ }^{15}$ and Kharb et al., ${ }^{16}$ which were similar to our study.

Homocysteine and migraine: A narrative review conducted by Lippi et al., ${ }^{10}$ found that in 5 of the 14 cross-sectional studies that measured plasma homocysteine levels, the concentration of plasma homocysteine was found to be significantly higher in migraine patients than in controls andthe concentration of plasma homocysteine was not found to be significantly higher in migraine patients than in controls in remaining 9 studies. In all four trials the treatment of hyperhomocysteinemia with folic acid (Vitamin B9) or vitamin B supplementation was effective to lower the plasma values of this biomarker, and was also associated with a decreased frequency and/or severity of migraine headache attacks. This association remains largely controversial, however, since it could not be clearly demonstrated that these two biological effects were directly linked. It is also noteworthy that vitamin B and folic acid may have some beneficial effect on 
migraine on the basis of serum homocysteine having a role of migraine pathogenesis. In our study we found serum homocysteine level is significantly higher in migraine patients than in controls. Hence, Vitamin B and Folic acid supplementation may be a possible prophylactic and therapeutic approach.

\section{Conclusion:}

The study suggests that the mean serum homocysteine level in migraineurs was significantly higher than that of non-migraineurs. The mean value of serum homocysteine level in female migraine patients was found increased than female control group which was statistically significant. The mean serum homocysteine level in male migraine was significantly higher than that of male control group. The mean serum homocysteine level in migraine patients without aura was significantly higher than in migraine patients with aura. Migraine headache severity and frequency of migraine attack were not affected by serum homocysteine level.

\section{References:}

1. Mohammad QD. Headache-A symptom not a Disease. J Bangladesh CollPhysSurg; (2013); 31:204-208.

2. Peter JG and Neil HR. 'Migraine and other primary headache Disorders'. In: Kasper DL, Hauser SL, Jameson JL, Fauci AS, Longo DL, Loscalzo J. Harrison's Principles of Internal Medicine. $19^{\text {th }}$ ed.; New York; McGraw-Hill Education;(2015): 2586-93.

3. Headache Classification Committee of the International Headache Society (HCCIHS). The International Classification of Headache Disorders, 3nd Edition (ICHD-3 beta version). Cephalalgia; (2013); 33 (9): 629-808.

4. Charles A. Advances in the Basic and Clinical Science of Migraine. Ann Neurology; (2009); 65: 491-98.

5. Bahadir A, Eroz R and Dikici S. Investigation of MTHFR C677T Gene Polymorphism, Biochemical and Clinical Parameters in Turkish Migraine Patients: Association with Allodyniaand Fatigue. Cell MolNeurobiol; () Springer Science + Business New York; (2013); 1-9.

6. Ropper $\mathrm{AH}$ and Brown $\mathrm{RH}$. 'Headache and other craniofacial pains' In: Samuels MA, Klein
JP and Allan HR. Adams and Victor's Principles of Neurology.' 10th ed.;NewYork; McGraw-Hill Education; (2014): 172-83.

7. Garza I, Todd JS, Smith JH and Robertson CE. Headache and other craniofacial pains. In: Daroff RB, Pomeroy SL, Jankovic J, Mazziotta JC. Bradley's Neurology in clinical practice. 7th ed.; Vol. (II); Philadelphia; Elsevier Saunders; (2016): 1695-1708.

8. Hannan MA, Hasan MK, Begum A, Haque A, Ullah AKM and Khan RK. Study of epidemiological features of primary headache patients in a tertiary center in Bangladesh. Bangladesh Journal of Neurosciences; (2007); 23: 13-20.

9. Moskowitz MA, (1984).The neurobiology of vascular head pain. Ann Neurol, 16: 157-168.

10. Lippi G, Mattiuzzi C, Meschi T, Cervellin G and Borghi L. Homocysteine and migraine. A narrative review. ClinicaChimicaActa; (2014); 433: 5-11.

11. Gavgani CS and Hoseinian MM. Comparative study on homocysteine level in migraine patients and normal peoples. Annals of Biological Research; (2012); 3(4): 1804-07.

12. Zhang X, Hong L, Haoli J, Ebin Z, Brodsky S and Goligorsky MS. Effects of homocysteine on endothelial nitric oxide production. Am. J. Physiol. Ren. Physiol;(2000); 279: 671-78.

13. Pizza V, Agresta A, Cassano D, Coluccid'Amato and Capasso A. The role of homocysteine in the pathogenesis of migrane. Current Neurobiology; (2013); 4(1\&2): 19-24.

14. Bokhari FA, Shakori TA, Hasan SAA, Qureshi $\mathrm{HJ}$ and Qureshi GA. Plasma homocysteine in patients of migraine without aura. J Ayub Med Coll Abbottabad; (2010); 22(2): 52-5.

15. Kharb N, Malik PK, Rani A. A cross-sectional study to detect the prevalence of hyperhomocysteinemia in patients of migraine. J. Evid. Based Med. Health.(2017); 4(78): 4614-21.

16. Sadeghi O, Maghsoudi Z, Askari G. Association between serum levels of homocysteine with characteristics of migraine attacks in migraine with aura. J Res Med Sci; (2014); 19(11): 1041-45. 